Advances in Theoretical and Applied Mathematics

ISSN 0973-4554 Volume 12, Number 2 (2017), pp. 65-69

(C) Research India Publications

https://dx.doi.org/10.37622/ATAM/12.2.2017.65-69

\title{
Some Expansion Formulae for the $\bar{H}$-Function
}

\author{
Yashwant Singh $^{1}$ and Nanda Kulkarni ${ }^{2}$ \\ ${ }^{1}$ Department of Mathematics, Government College, Kaladera, Jaipur (Rajasthan), \\ India
}

${ }^{2}$ Department of Mathematics, Maharani Lakshmi Ammanni College for Women Bangalore, Karnataka, India

\begin{abstract}
In the present paper, the authors have established two expansion formula of $\bar{H}$ -Function.
\end{abstract}

Key words: $\bar{H}$-Function, Expansion Formula, Gamma Function

(2000 Mathematical Subject Classification: 33C99)

\section{INTRODUCTION}

The $\bar{H}$-function occurring in the paper will be defined and represented as follows:

$$
\bar{H}_{P, Q}^{M, N}[z]=\bar{H}_{P, Q}^{M, N}\left[z \mid \begin{array}{c}
\left(a_{j} ; \alpha_{j} ; a_{j}\right)_{1, N},\left(a_{j} ; \alpha_{j}\right)_{N+1, P} \\
\left(b_{j}, \beta_{j}\right)_{1, M},\left(b_{j}, \beta_{j} ; B_{j}\right)_{M+1, Q}
\end{array}\right]=\frac{1}{2 \pi i} \int_{-i \infty}^{i \infty} \bar{\phi}(\xi) z^{\xi} d \xi
$$

where $\quad \bar{\phi}(\xi)=\frac{\prod_{j=1}^{M} \Gamma\left(b_{j}-\beta_{j} \xi\right) \prod_{j=1}^{N}\left\{\Gamma\left(1-a_{j}+\alpha_{j} \xi\right)\right\}^{A_{j}}}{\prod_{j=M+1}^{Q}\left\{\Gamma\left(1-b_{j}+\beta_{j} \xi\right)\right\}^{B_{j}} \prod_{j=N+1}^{P} \Gamma\left(a_{j}-\alpha_{j} \xi\right)}$ 
Which contains fractional powers of the gamma functions. Here, and throughout the paper $a_{j}(j=1, \ldots, p) \quad$ and $\quad b_{j}(j=1, \ldots, Q)$ are complex parameters, $\alpha_{j} \geq 0(j=1, \ldots, P), \beta_{j} \geq 0(j=1, \ldots, Q)$ (not all zero simultaneously) and exponents $A_{j}(j=1, \ldots, N)$ and $B_{j}(j=N+1, \ldots, Q)$ can take on non integer values.

The following sufficient condition for the absolute convergence of the defining integral for the $\bar{H}$-function given by equation (1.1) have been given by (Buschman and Srivastava[1]).

$\Omega \equiv \sum_{j=1}^{M}\left|\beta_{j}\right|+\sum_{j=1}^{N}\left|A_{j} \alpha_{j}\right|-\sum_{j=M+1}^{Q}\left|\beta_{j} B_{j}\right|-\sum_{j=N+1}^{P}\left|\alpha_{j}\right|>0$

and $|\arg (z)|<\frac{1}{2} \pi \Omega$

If we take $A_{j}=1(j=1, \ldots, N), B_{j}=1(j=M+1, \ldots, Q)$ in (1.1), the function $\bar{H}_{P, Q}^{M, N}$ reduces to the Fox's H-function [2].

We shall use the following notation:

$$
A^{*}=\left(a_{j}, \alpha_{j} ; A_{j}\right)_{1, N},\left(a_{j}, \alpha_{j}\right)_{N+1, P}, B^{*}=\left(b_{j}, \beta_{j}\right)_{1, M},\left(b_{j}, \beta_{j} ; B_{j}\right)_{M+1, Q},
$$

\section{EXPANSION FORMULA}

\section{First Formula}

$$
\bar{H}_{p, q}^{m, n}\left[\left.\eta \omega\right|_{B^{*}} ^{A^{*}}\right]=\eta^{\frac{\left(a_{1}-1\right)}{\alpha_{1}}} \sum_{r=0}^{\infty} \frac{\left[1-\eta^{1 / \alpha_{1}}\right]^{r}}{r !} \bar{H}_{p, q}^{m, n}\left[\left.\omega\right|_{B^{*}} ^{\left(-r+a_{1}, \alpha_{1} ; 1\right),\left(a_{j}, \alpha_{j} ; A_{j}\right)_{2, n},\left(a_{j}, \alpha_{j}\right)_{n+1, p}}\right]
$$

Where $\eta>0, \operatorname{Re}\left(\eta^{1 / \alpha}\right)>\frac{1}{2} ; \arg (\eta \omega)=\alpha_{1} \arg \left(\eta^{1 / \alpha_{1}}\right)+\arg \omega$ and $\left|\arg \left(\eta^{1 / \beta_{1}}\right)\right|<\frac{\pi}{2}$.

Proof: R.H.S. $=\eta^{\frac{\left(a_{1}-1\right)}{\alpha_{1}}} \sum_{r=0}^{\infty} \frac{\left[1-\eta^{1 / \alpha_{1}}\right]^{r}}{r !} \bar{H}_{p, q}^{m, n}\left[\left.\omega\right|_{B^{*}} ^{\left(-r+a_{1}, \alpha_{1} ; 1\right),\left(a_{j}, \alpha_{j} ; A_{j}\right)_{2, n},\left(a_{j}, \alpha_{j}\right)_{n+1, p}}\right]$ 


$$
\eta \sum_{r=0}^{\left(a_{1}-1\right) / \alpha_{1}} \sum^{\infty} \frac{\left[1-\eta^{1 / \alpha_{1}}\right]^{r}}{r !} \frac{1}{2 \pi i} \int_{L} \frac{\Gamma\left(1+r-a_{1}+\alpha_{1} s\right) \prod_{j=1}^{m} \Gamma\left(b_{j}-\beta_{j} s\right) \prod_{j=2}^{n}\left\{\Gamma\left(1-a_{j}+\alpha_{j} s\right)\right\}^{A_{j}}}{\prod_{j=M+1}^{q}\left\{\Gamma\left(1-b_{j}+\beta_{j} s\right)\right\}^{B_{j}} \prod_{j=N+1}^{p} \Gamma\left(a_{j}-\alpha_{j} s\right)} \omega^{s} d s
$$

Changing the order of integration and summation under the integral sign

$=\frac{1}{2 \pi i} \int_{L} \bar{\phi}(s) \omega^{s}\left[\eta^{\left(a_{1}-1\right) / \alpha_{1}} \sum_{r=0}^{\infty} \frac{\left[1-\eta^{1 / \alpha_{1}}\right]^{r}}{r !} \Gamma\left(1+r-a_{1}+\alpha_{1} s\right)\right] d s$

Where $\bar{\phi}(s)=\frac{\prod_{j=2}^{m} \Gamma\left(b_{j}-\beta_{j} s\right) \prod_{j=2}^{n}\left\{\Gamma\left(1-a_{j}+\alpha_{j} s\right)\right\}^{A_{j}}}{\prod_{j=m+1}^{q}\left\{\Gamma\left(1-b_{j}+\beta_{j} s\right)\right\}^{B_{j}} \prod_{j=n+1}^{p} \Gamma\left(a_{j}-\alpha_{j} s\right)}$

$=\eta\left(a_{1}-1\right) / \alpha_{1} \sum_{r=0}^{\infty} \frac{\left[1-\eta^{1 / \alpha_{1}}\right]^{r}}{r !} \frac{1}{2 \pi i} \int_{L} \bar{\phi}(s) \omega^{s}\left(1-a_{1}+\alpha_{1} s\right)_{r} \Gamma\left(1-a_{1}+\alpha_{1} s\right) d s$

$=\eta^{\left(a_{1}-1\right) / \alpha_{1}} \frac{1}{2 \pi i} \int_{L} \bar{\phi}(s) \omega^{s}\left[1-1+\eta^{1 / \alpha_{1}}\right]^{\left.-1+a_{1}-\alpha_{1} s\right)} \Gamma\left(1-a_{1}+\alpha_{1} s\right) d s$

$$
\left[\therefore \sum \frac{x^{r}}{r !}(a)_{r}=(1-x)^{-a}\right]
$$

$=\eta^{\left(a_{1}-1\right) / \alpha_{1}} \frac{1}{2 \pi i} \int_{L} \bar{\phi}(s) \omega^{s} \eta^{-\left(a_{1}-1\right) / \alpha_{1}} \eta^{s} \quad \Gamma\left(1-a_{1}+\alpha_{1} s\right) d s$

$=\frac{1}{2 \pi i} \int_{L} \bar{\phi}(s) \omega^{s} \eta^{s} \Gamma\left(1-a_{1}+\alpha_{1} s\right) d s$

$=\frac{1}{2 \pi i} \int_{L} \frac{\prod_{j=1}^{m} \Gamma\left(b_{j}-\beta_{j} s\right) \prod_{j=1}^{n}\left\{\Gamma\left(1-a_{j}+\alpha_{j} s\right)\right\}^{A_{j}}}{\prod_{j=M+1}^{q}\left\{\Gamma\left(1-b_{j}+\beta_{j} s\right)\right\}^{B_{j}}}(\omega \eta)^{s} d s=$ L.H.S. 


\section{Second Formula}

$\bar{H}_{p, q}^{m, n}\left[\left.\eta \omega\right|_{B^{*}} ^{A^{*}}\right]=\eta^{\frac{\left(a_{p}-1\right)}{\alpha_{p}}} \sum_{r=0}^{\infty} \frac{\left[1-\eta^{1 / \alpha_{p}}\right]^{r}}{r !} \bar{H}_{p, q}^{m, n}\left[\left.\omega\right|_{B^{*}} ^{\left(a_{j}, \alpha_{j} ; A_{j}\right)_{1, n},\left(a_{j}, \alpha_{j}\right)_{n+1, p-1},\left(-r+a_{p}, \alpha_{p}\right)}\right]$

Where $p>n, \operatorname{Re}\left(\eta^{1 / \alpha_{p}}\right)>\frac{1}{2} ; \arg (\eta \omega)=\alpha_{p} \arg \left(\eta^{1 / \alpha_{p}}\right)+\arg \omega$ and $\left|\arg \left(\eta^{1 / \beta_{1}}\right)\right|<\frac{\pi}{2}$.

Proof: R.H.S. $=\eta^{\frac{\left(a_{p}-1\right)}{\alpha_{p}}} \sum_{r=0}^{\infty} \frac{\left[1-\eta^{1 / \alpha_{p}}\right]^{r}}{r !} \bar{H}_{p, q}^{m, n}\left[\left.\omega\right|_{B^{*}} ^{\left(a_{j}, \alpha_{j} ; A_{j}\right)_{1, n},\left(a_{j}, \alpha_{j}\right)_{n+1, p-1},\left(-r+a_{p}, \alpha_{p}\right)}\right]$

$$
\eta\left(a_{p}-1\right) / \alpha_{p} \sum_{r=0}^{\infty} \frac{\left[1-\eta^{1 / \alpha_{p}}\right]^{r}}{r !} \frac{1}{2 \pi i} \int_{L} \frac{\prod_{j=1}^{m} \Gamma\left(b_{j}-\beta_{j} s\right) \prod_{j=2}^{n}\left\{\Gamma\left(1-a_{j}+\alpha_{j} s\right)\right\}^{A_{j}}}{\Gamma\left(-r+a_{p}-\alpha_{p} s\right) \prod_{j=M+1}^{q}\left\{\Gamma\left(1-b_{j}+\beta_{j} s\right)\right\}^{B_{j}} \prod_{j=N+1}^{p} \Gamma\left(a_{j}-\alpha_{j} s\right)} \omega^{s} d s
$$

Changing the order of integration and summation under the integral sign

$$
=\eta\left(a_{p}-1\right) / \alpha_{p} \sum_{r=0}^{\infty} \frac{\left[1-\eta^{1 / \alpha_{p}}\right]^{r}}{r !} \frac{1}{2 \pi i} \int_{L} \bar{\phi}(s) \omega^{s} \frac{1}{\Gamma\left(-r+a_{p}-\alpha_{p} s\right)} d s
$$

Where $\bar{\phi}(s)=\frac{\prod_{j=2}^{m} \Gamma\left(b_{j}-\beta_{j} s\right) \prod_{j=1}^{n}\left\{\Gamma\left(1-a_{j}+\alpha_{j} s\right)\right\}^{A_{j}}}{\prod_{j=m+1}^{q}\left\{\Gamma\left(1-b_{j}+\beta_{j} s\right)\right\}^{B_{j}} \prod_{j=n+1}^{p-1} \Gamma\left(a_{j}-\alpha_{j} s\right)}$

$=\eta\left(a_{p}-1\right) / \alpha_{p} \frac{1}{2 \pi i} \int_{L} \bar{\phi}(s) \omega^{s} \sum_{r=0}^{\infty} \frac{\left[1-\eta^{1 / \alpha_{p}}\right]^{r}}{r !} \times \frac{1}{\left(a_{p}-\alpha_{p} s\right)_{r} \Gamma\left(a_{p}-\alpha_{p} s\right)} d s$

$=\eta^{\left(a_{p}-1\right) / \alpha_{p}} \frac{1}{2 \pi i} \int_{L} \bar{\phi}(s) \omega^{s} \frac{1}{\Gamma\left(a_{p}-\alpha_{p} s\right)} \sum_{r=0}^{\infty} \frac{\left[\eta^{1 / \alpha_{p}}-1\right]^{r}}{r !} \frac{\left(1-a_{p}+\alpha_{p} s\right)_{r}}{(-1)^{r}} d s$

$$
\left[\therefore \sum \frac{x^{r}}{r !}(a)_{r}=(1-x)^{-a}\right]
$$




$$
\begin{aligned}
& =\eta^{\left(a_{p}-1\right) / \alpha_{p}} \frac{1}{2 \pi i} \int_{L} \bar{\phi}(s) \omega^{s} \frac{1}{\Gamma\left(a_{p}-\alpha_{p} s\right)} \frac{\left(1-\eta^{-1 / \alpha_{p}}\right)^{r}}{r !}\left(1-a_{p}+\alpha_{p} s\right)_{r} d s \\
& =\eta^{\left(a_{p}-1\right) / \alpha_{p}} \frac{1}{2 \pi i} \int_{L} \bar{\phi}(s) \omega^{s} \frac{1}{\Gamma\left(a_{p}-\alpha_{p} s\right)} \eta^{-\left(a_{p}-1\right) / \alpha_{p}} \eta^{s} d s \\
& =\frac{1}{2 \pi i} \int_{L} \bar{\phi}(s) \omega^{s} \eta^{s} \frac{1}{\Gamma\left(a_{p}-\alpha_{p} s\right)} d s \\
& =\frac{1}{2 \pi i} \int_{L} \frac{\prod_{j=1}^{q} \Gamma\left(b_{j}-\beta_{j} s\right) \prod_{j=1}^{n}\left\{\Gamma\left(1-a_{j}+\alpha_{j} s\right)\right\}^{A_{j}}}{\prod_{j=M+1}^{q}\left\{\Gamma\left(1-b_{j}+\beta_{j} s\right)\right\}^{B_{j}} \prod_{j=N+1}^{p} \Gamma\left(a_{j}-\alpha_{j} s\right)}(\omega \eta)^{s} d s=\text { L.H.S. }
\end{aligned}
$$

For $A_{j}=1(j=1, \ldots, n), B_{j}=1(j=m+1, \ldots, q)$ in (2.1),(2.2), we get the results in terms of Fox's H-function [2].

\section{REFERENCES}

[1] Buschman, R.G. and Srivastava , H.M.; The $\bar{H}$ - function associated with a certain class of Feynman integrals, J.Phys.A:Math. Gen. 23, (1990), 47074710

[2] Fox, C.; The $G$-function and $H$ function as symmetric Fourier kernels, Trans. Amer. Math. Soc. 98, (1961), 396-429.

[3] Srivastava,H.M.,Gupta, K.C. and Goyal,S.P.; The H-Functions of One and Two Varibale with Applications,South Asian Publishers, New Delhi and Madras, (1982). 
